\title{
MULTIDIREKTIONALE FORMEN DES ERINNERNS UND VERGESSENS. DAS BEISPIEL EINER POSTKOLONIALEN UND POSTSOZIALISTISCHEN STADTFÜHRUNG
}

Miriam Friz Trzeciak

Brandenburgische Technische Universität Cottbus - Senftenberg,

Lehrstuhl Interkulturalität

E-Mail: Miriam.Trzeciak@b-tu.de

URL: https://www.b-tu.de/fg-interkulturalitaet/team/akademisches-team/dr-miriam-

$\underline{\text { friz-trzeciak }}$

Zitationsvorschlag:

Trzeciak, Miriam Friz (2020): Multidirektionale Formen des Erinnerns und Vergessens. Das Beispiel einer postkolonialen und postsozialistischen Stadtführung. In: Gesellschaft - Individuum - Sozialisation (GISo). Zeitschrift für Sozialisationsforschung, 1 (2). DOI: 10.26043/GISo.2020.2.4

Link zum Artikel:

https://doi.org/10.26043/GISo.2020.2.4 


\title{
MULTIDIREKTIONALE FORMEN DES ERINNERNS UND VERGESSENS. DAS BEISPIEL EINER POSTKOLONIALEN UND POSTSOZIALISTISCHEN STADTFÜHRUNG
}

\author{
Miriam Friz Trzeciak
}

\begin{abstract}
Die Bewegungsgeschichte migrantischer und BIPoC communities in Ostdeutschland wird genauso wie das koloniale Erbe der Städte im kollektiven Gedächtnis der BRD weiterhin vernachlässigt. Das Ausblenden dieser Geschichten ist nicht nur auf postsozialistische Prozesse der Abwertung ostdeutscher Erfahrungen zurückzuführen, auch die DDR imaginierte sich als weiße Nation und reproduzierte postkoloniale Prozesse des Fremdmachens. Um vergessene und entinnerte Geschichten sichtbar zu machen, greift der Beitrag auf die Befunde der memory studies sowie auf die post- und dekoloniale Theoriebildung zurück. Aufbauend auf den Erkenntnissen, die im Rahmen des Lehrforschungsprojektes einer postkolonialen und postsozialistischen Stadtführung gewonnen wurden, gibt der Beitrag Impulse zum Überdenken der Verflechtungen zwischen Sozialismus und Kolonialismus. Aufbauend auf Michael Rothbergs (2009) Überlegungen zu multidirectional memory argumentiert der Beitrag, dass verschiedene Formen der Erinnerung nicht kompetitiv, sondern dialogisch wirken können. Über multidirektionale Formen der Erinnerung können Gegennarrative zu rechtsextremen und rassistischen Narrativen hergestellt werden.
\end{abstract}

Keywords: DDR, postkoloniale Perspektiven, Postsozialismus, multi-sited ethnography, dekoloniale Stadtführung, dialogische Forschung, multidirectional memory

\section{EINLEITUNG}

Die ostdeutsche Stadt Cottbus wird seit Januar 2017 in den Medien als emblematischer Ort von Konflikten um Migration verhandelt. ${ }^{1}$ Nach einer gewaltsamen Auseinandersetzung zwischen deutschen und syrischen Jugendlichen geriet die mittelgroße Stadt in die Schlagzeilen (Trzeciak/Schäfer 2019). Viele Medien berichteten sehr polarisierend über die Ereignisse. In einigen Darstellungen schien es, als sei die Stadtgesellschaft in drei stereotype Lager aufgeteilt: in rechtsextreme Hooligans, verunsicherte Bürger*innen sowie gewaltbereite und bedrohliche Geflüchtete (ebd.). In der Tat hat die Stadt - wie viele andere Städte in Deutschland - ein Problem mit Rassismus. Im bundesweiten Vergleich sticht sie sogar mit beunruhigenden Tendenzen hervor: Die AfD wurde in der Landtagswahl im
September 2019 mit $26.8 \%$ der Stimmen die stärkste Kraft in Cottbus (Stadtverwaltung Cottbus 2019). Parallel zur demokratischen Legitimierung rechter und rechtsextremer Positionen haben seit 2015 rassistisch motivierte Gewalttaten in der Stadt in besorgniserregender Weise zugenommen. ${ }^{2}$

Für den spezifischen Rassismus und Rechtsextremismus in Ostdeutschland lassen sich aus historischer Perspektive „konkret rekonstruierbare Ursachen" (Behrends et al. 2003, 14) angeben zentral sind $u$. a. das Fehlen einer starken Zivilgesellschaft sowie die Leugnung der Existenz von Rassismus durch die SED-Führung in der DDR. Die medialen Darstellungen heute simplifizieren allerdings häufig die soziale Realität und bedienen ausgehend von den einzelnen Ereignissen

\footnotetext{
${ }^{1}$ Ich danke Judith Lippelt, Christian Obermüller, Franziska Müller und den anonymen Gutachter*innen für hilfreiche Anmerkungen. Frühere Überlegungen zu diesem Projekt haben Manuel Peters und ich (i. E.) an anderer Stelle ausgeführt.

2 Der Verein Opferperspektive (2019), der im Land Brandenburg Betroffene von rechter Gewalt und rassistischer Diskriminierung berät, zählte 2019 insgesamt 14 und 201835 rechte Gewalttaten in Cottbus.
} 
essentialisierende und rassistische Deutungsmuster.

In der Berichterstattung über Cottbus kommen neben rassistischen Stereotypen wie dem Bild des sexuell aggressiven männlichen Geflüchteten auch räumlich-zeitlich kulturalisierende Topoi zum Tragen (Heft 2018). Dabei wird Ostdeutschland im Verhältnis zu einem vermeintlich progressiveren Westdeutschland als weniger zivilisiert und demokratisch dargestellt. Dies ist beispielsweise der Fall, wenn Rassismus als gesamtgesellschaftliches Problem auf den „braunen" Osten (ebd.; Kollmorgen/Hans 2011) verlagert und koloniale (Dis)Kontinuitäten von Rassismus im Kontext einer postkolonialen und postnationalsozialistischen Gesellschaft ausgeblendet werden (Messerschmidt 2008).

Wie aber können alternative, vergessene und marginalisierte Geschichten im kollektiven Gedächtnis der Stadtgesellschaft sichtbar gemacht werden? Und wie können auf diese Weise Grundlagen für multidirektionale und dialogische Formen der Erinnerung geschaffen werden?

Im Folgenden werde ich am Beispiel der Entwicklung einer postkolonialen und postsozialistischen Stadtführung in Cottbus eine kollaborative und dialogische Methode zur Rekonstruktion und Reflexion im kollektiven Gedächtnis marginalisierter Geschichten in der Stadtgesellschaft aufzeigen. In einem ersten Schritt gebe ich einen Überblick über verschiedene Etappen der Einwanderung in Cottbus. In einem zweiten Schritt greife ich auf die Befunde der memory studies zurück und zeige, dass die Wissensbestände des kollektiven Gedächtnisses einer (Stadt-)Gesellschaft auf machtvolle und umkämpfte Prozesse der Konstruktion von Erinnerung und Zugehörigkeit verweisen. Mit Michael Rothberg (2009) plädiere ich für nicht-kompetitive, d.h. kooperierende, und multidirektionale Formen des Erinnerns, für multidirectional memory. Diese Überlegungen nehme ich zum Ausgangspunkt, um in einem dritten Schritt am Beispiel des Lehrforschungsprojekts einer postkolonialen und postsozialistischen Stadtführung eine dialogische und kollaborative Methode der Geschichtsaufarbeitung vorzustellen, um marginalisierte oder vergessene Geschichten in Cottbus sichtbar zu machen. Anhand von zwei konkreten
Erinnerungsorten gebe ich in einem vierten Schritt einen Ausblick auf die erinnerungspolitischen Aushandlungsprozesse, die im Kontext der Stadtführung stattgefunden haben. Ich argumentiere, dass die Prozesse der gemeinsamen Erinnerungsarbeit nicht nur auf verwobenen postkolonialen und postsozialistischen Machtkonstellationen beruhen, sondern dass die dialogische Methode der Stadtführung vielmehr einen Raum für ambivalente, sich überlagernde Formen der multidirektionalen Erinnerung herstellen kann. Ich schließe mit Überlegungen dazu, dass die Aufarbeitung von postkolonialen und postsozialistischen Spuren ostdeutscher Städte dazu beitragen kann, rassistischen und rechtsextremen Narrativen etwas entgegenzusetzen.

\section{MULTIDIREKTIONALE ERINNERUNG}

Wie die memory studies (Rothberg 2009; Assman 2018) aufgezeigt haben, kann Erinnerung als Werkzeug verstanden werden, mit dessen Hilfe das Vergangene in der Gegenwart verhandelt wird. Jede Gesellschaft hat eine spezifische Beziehung zu ihrer Vergangenheit (Harvey 2001, 320). Die Frage, wie diese Vergangenheit erzählt wird, verweist auf die Konstruktion einer „national story“, die festlegt, wer zu einer Gesellschaft zählt und als erinnerungswürdig gilt (Hall 1999, 4). Die Aushandlungen von Vergangenheitsnarrativen, wie sie etwa in nationalen $\mathrm{Mu}$ seen oder Gedenkveranstaltungen zum Tragen kommen, stehen im Zusammenhang hegemonialer Kämpfe um die Konstruktion einer offiziellen Geschichtsschreibung. Sie beeinflussen die Möglichkeiten gesellschaftlicher Teilhabe und adressieren Fragen von Identität und Zugehörigkeit (Rothberg 2009, 5; Rothberg/Yildiz 2011).

Erinnerungen können dementsprechend als symbolische Repräsentationen der Vergangenheit verstanden werden, die in soziale Handlungen eingebunden sind (Confino/Fritzsche 2002, 5). Sie müssen aktiv und teils entgegen erheblicher gesellschaftlicher Widerstände durch Praktiken oder Interventionen erarbeitet werden. Erst über diese Formen der Arbeit werden Erinnerungen als sozial bedeutsam hergestellt (Rothberg 2009, 4). Folglich bezeichnet Erinnerung kein neutrales oder zwangsläufiges Unterfangen, sondern vielmehr einen Prozess, der sich sowohl auf die verkörperten und gelebten als auch auf 
die sozialen und fabrizierten Aspekte der Beziehung von Individuen, Gruppen und Gesellschaften zur Vergangenheit bezieht (ebd.). ${ }^{3}$ Demnach sagt die Art und Weise, wie und ob Menschen und ihre Praktiken erinnert werden, mehr über die gegenwärtigen sozialen Konstellationen und Verhältnisse aus als über die faktischen Geschehnisse in der Vergangenheit.

Auch wenn es sich bei Erinnerungen um machtvolle Artikulationen handelt, die auf Kämpfe um Deutungsmacht und Anerkennung hinweisen, so argumentiert Michael Rothberg (ebd., 4 ff.), dass dies nicht zwangsläufig in einer kompetitiven Logik resultieren muss. Mit seiner programmatischen Arbeit zu Formen von multidirectional memory (multidirektionales Gedächtnis/Erinnerung), hinterfragt er die in den memory studies gängige Verbindung zwischen kollektiver Erinnerung und Gruppenidentität. An der Schnittstelle von postkolonialer Forschung und holocaust studies hebt er die multidirektionalen und transnationalen Aspekte von Erinnerungen und kollektivem Gedächtnis hervor. Rothberg zeigt, wie postkoloniale Autor*innen (wie Aimé Césaire oder W. E. B. Du Bois) das Gedenken an den Holocaust produktiv machen und subversiv nutzen, um daran anknüpfend das Erbe von KoIonialismus und Versklavung sowie die Notwendigkeit einer Dekolonisierung zu thematisieren (ebd., xiii). Die Erinnerung an den Holocaust dient als Deckerinnerung (screen memory), um andere Formen der gewaltvollen, genozidalen und traumatisierenden Gewalt in der Vergangenheit in Erinnerung zu rufen und geltend zu machen. Die Arenen, in denen Erinnerungen zu sich überkreuzenden historischen Hintergründen verhandelt werden, versteht Rothberg (ebd.) somit als diskursive Räume, in denen verschiedene Gruppen über vergangenheitsbezogene Dialoge und Interaktionen in Erscheinung treten. Aus dieser Sichtweise können Erinnerungen als anhaltende Prozesse der Verhandlung, des Quer-Verweisens und des Ausleihens beschrieben werden. Die Aufarbeitung spezifischer Ereignisse in der
Vergangenheit seitens verschiedener sozialer Gruppen kann als eine Kontakt-Zone fungieren, entlang derer sich transnationale kulturelle Räume aufspannen (ebd.). Durch die produktiven, transkulturellen und öffentlichen Eigenschaften birgt multidirectional memory das Potential, die Logik der Konkurrenz um Anerkennung zwischen verschiedenen sozialen Gruppen zu durchbrechen und auf diese Weise Räume für verschiedene Formen der Erinnerung, aber auch neue Formen der Solidarität und neue Visionen von Gerechtigkeit zu schaffen (ebd., 3). Indem multidirectional memory die Beziehungen zwischen Minderheiten sowie marginalisierten und hegemonialen Erinnerungen in den Vordergrund stellt, adressiert das Konzept die produktiven und überraschenden Begegnungen im Kontext von (asymmetrischen) Aushandlungen von Erinnerung (Attia/Rothberg 2018, 98).

\section{MIGRATION IN DER POSTKOLONIALEN UND POSTSOZIALISTISCHEN STADT}

Um die (Dis-)Kontinuitäten des Rassismus in Cottbus besser verstehen zu können, müssen diese Geschichten und Erfahrungen in den Zusammenhang der Prozesse des Fremdmachens und der Rassialisierung in einer postfaschistischen, postkolonialen und postsozialistischen Gesellschaft gesetzt werden (El-Tayeb 2016, $37 \mathrm{ff}$.$) . Hier schließe ich an die Arbeiten aus der$ postkolonialen Forschung zu Erinnerungspolitik an, die sowohl für die Herstellung neuer Zukunftsvisionen als auch für die Erarbeitung neuer Vergangenheitsnarrative plädiert haben (ebd.; Ha, N. K. 2017; Ha, K. N. 2017). Weiter argumentiere ich mit einer sozialkonstruktivistischen Perspektive, dass Migration in ihrer Bedeutsamkeit erst im Zuge gesellschaftlicher Machtkonstellationen als machtvolles Bündel von Praktiken und Diskursen produziert wird. Die Prozesse des doing migration (Amelina 2017) sind auch dann wirkmächtig, wenn Formen bereits vergangener Zugehörigkeit und Teilhabe in der Gegenwart verhandelt und auf diese Weise ungleich

\footnotetext{
3 Jan Assmann (1999) und Aleida Assmann (2018) differenzieren in ihren Überlegungen zum kollektiven Gedächtnis zwischen einem kommunikativen und einem kulturellen Gedächtnis. Während das kommunikative Gedächtnis auf der mündlichen Übermittlung von Erinnerungen basiert, wird das kulturelle Gedächtnis über Dokumente, d. h. niedergeschriebene Erinnerungen, vermittelt (ebd.). Ist das kommunikative Gedächtnis auf Personen angewiesen und erlischt nach drei Generationen, so konservieren schriftliche Zeugnisse kollektive Erinnerungen und sind an der Konstruktion eines nationalen Gedächtnisses beteiligt.
} 
gelagerte Formen der Aus- und Abgrenzung von inferior imaginierten Anderen in der Gegenwart geschaffen werden (El-Tayeb 2016).

Die Geschichten von marginalisierten, rassialisierten und fremd-gemachten Gruppen (z. B. migrantischen und/oder BIPoC communities eine politische Selbstbezeichnung für Black, Indigenous und People of Color) sind im kollektiven Gedächtnis nur wenig präsent, oftmals werden sie von hegemonialen und essentialistischen Narrativen der Nation als Volk mit einer gemeinsamen Geschichte, Sprache und Kultur überlagert (Attia/Rothberg 2018, 100). In der Cottbuser Stadtgesellschaft sind diese Geschichten sowie die rassistischen (Dis-)Kontinuitäten von Ausgrenzung und Gewalt ebenfalls selten zu hören. Auch wenn die DDR im Vergleich zur BRD ihre koloniale und imperialistische Vergangenheit teilweise kritischer und aktiver aufarbeitete, so machten rassistische Bilder und Praktiken, die aus den Ideologien des Kaiserreichs, der Weimarer Republik und des NS-Regimes resultierten, keinen Halt vor den Grenzen der DDR (Mende 2013, 162; Ha, K. N. 2017. 114 f.). Die staatlich propagierten Politiken des Antiimperialismus und Antifaschismus sorgten dafür, dass koloniale und rassistische Denkmuster unproblematisiert blieben, da sie offiziell nicht existierten (Poutrus 2005a, 2 ff.; Behrends et al. 2003).

Wie an vielen anderen Orten sind BIPoC Teil der Stadtgeschichte von Cottbus. Zwar hat sich das Stadtbild mit dem Ankommen von Geflüchteten nach 2015 verändert und das Thema Migration scheint in der Stadt präsenter zu sein. Aber bereits eine oberflächliche Recherche gibt Hinweise darauf, dass Cottbus eine kontinuierliche Migrationsgeschichte aufweist. So kam es im Zuge der brutalen Expeditionen und Expansionen der Kolonialmacht Brandenburg im heutigen Ghana zur gewaltvollen Verschleppung und Versklavung von Bewohner*innen dieser und angrenzender Regionen (Conrad 2008, 18 f.). Auch wenn der Großteil der versklavten Menschen in die Karibik gebracht wurde, so gelangten auch einige von ihnen, häufig als exotisierte Beschäftigte bei Hofe, nach Brandenburg-Preußen (Theilig 2013). Ein weiteres Beispiel für die unfreiwillige und unter gewaltvollen Bedingungen erfolgte Präsenz von BIPoC in der Region ist die Geschichte von Machbuba, die vermutlich aus dem heutigen Äthiopien stammte und die Hermann Ludwig Heinrich Fürst von Pückler-Muskau 1837 minderjährig auf einem Sklavenmarkt in Ägypten "kaufte" und nach Bad Muskau brachte (Schwachenwald 2019). Während des 1. Weltkrieges wurden Menschen später aus dem globalen Süden in einem Kriegsgefangenenlager in Cottbus interniert und für Propagandazwecke des Deutschen Reiches rassialisiert (etwa durch Darstellungen auf Postkarten; Dortmund Postkolonial 2016). In der Zeit des Nationalsozialismus wurden Jüd*innen, Kommunist*innen und weitere soziale Gruppen in der Stadt verfolgt und deportiert. Tausende Menschen wurden in den Konzentrationslagern der Region interniert und/oder als Zwangsarbeiter*innen ausgebeutet und ermordet (Häfner/Müller 2020).

Auch in der DDR gab es verschiedene Ein- und Auswanderungsbewegungen. Auch wenn aktivistische und akademische Arbeiten begonnen haben, die Erfahrungen migrantischer und diasporischer BIPoC communities während und nach 1989 aufzuarbeiten (Piesche 2019), ist das Bild eines natio-ethno-kulturell homogenen DDR-Staates im kollektiven Gedächtnis weiterhin vorherrschend (Goel 2013; Ha 2019). Diese Perspektive schreibt u. a. das auf kolonialen Epistemen beruhende Selbstverständnis der DDR als weiße ${ }^{4}$ Nation fort (Piesche 2002, 55). Zwar war mit Ausnahme der Angehörigen russischer Besatzungstruppen der Anteil von Ausländer*innen in der DDR-Bevölkerung vergleichsweise gering (Poutrus 2005a, 6). Während DDR-Bürger*innen massenhaft auswanderten, bildete die Einreise von Ausländer*innen insgesamt eine "extreme Ausnahme" (ebd.). ${ }^{5}$ Jedoch ist davon auszugehen, dass verschiedene Formen der Zuwanderung die sozialen Realitäten in

\footnotetext{
${ }^{4}$ Um auf die soziale Konstruktion sowie die hierarchisierende Wirkmächtigkeit von race und Prozessen der Rassialisierung hinzuweisen, schreibe ich weiß klein und kursiv. In Abgrenzung dazu kennzeichne ich Schwarz als politische Positionierung und Selbstbezeichnung durch Großschreibung (Eggers et al. 2005).

51989 betrug der Anteil von Ausländer*innen in der DDR-Bevölkerung 1.1 \%, in der westdeutschen Bevölkerung $7.7 \%$ (Goel 2013, 140 f.).
} 
industriellen und urbanen Räumen wie Cottbus, zu DDR-Zeiten ein bedeutendes Zentrum der Energie- und Textilwirtschaft, massiv prägten. Ab den 1980er-Jahren wurden im Zuge des Arbeitskräftemangels verstärkt vietnamesische Arbeitsmigrant*innen in der Cottbuser Textilproduktion eingesetzt (Strnad 2011). Weiter kamen einige der 2.000 politischen Exilant*innen nach dem Militärputsch in Chile 1973 nach Cottbus (Maurin 2005).

Je nachdem ob Menschen aus dem Exil (wie Griech*innen oder Chilen*innen), als Studierende (aus sozialistischen oder westlichen Ländern) oder als Vertragsarbeiter*innen (aus sozialistischen Staaten wie Vietnam, Mosambik, Angola, Kuba, Algerien oder Ungarn) in die DDR kamen, waren ihre Lebensrealitäten sehr unterschiedlich (Poutrus 2005a, 2005b). Den Umgang mit Migrant*innen in der DDR kennzeichnete, dass diese nicht über garantierte Rechte verfügten. So stellte Asyl eine Kann-Regelung dar, die letztendlich den Entscheidungen des Führungskaders der SED oblag (Poutrus 2005a, 9). Und auch wenn Vertragsarbeiter*innen - in den 1980erJahren die größte Gruppe von Ausländer*innen formal ihren deutschen Kolleg*innen in den Betrieben gleichgestellt waren, arbeiteten sie oftmals in den körperlich anstrengendsten und niedrigsten Arbeitsbereichen und waren einer Politik der "staatlich verordneten Abgrenzung" (ebd., 11) zu den DDR-Bürger*innen ausgesetzt. Zusammenfassend lässt sich sagen, dass Migration in der DDR unter sehr restriktiven Bedingungen stattfand und im Alltag umfassend kontrolliert wurde (Mende 2013, 151). Die Prozesse der Klassifizierung, die mit den unterschiedlichen Formen des Aufenthaltsstatus einhergingen, machten Migrant*innen zu Fremden und sorgten für marginalisierte Positionen sowie deren Stabilisierung (Poutrus 2005a, 14 f.).

Als die BRD nach 1990 die Migrationsabkommen und Verträge der DDR nicht übernahm, wurde ein Großteil der Vertragsarbeiter*innen arbeitslos und musste das Land verlassen. Einigen gelang es jedoch, den Kampf um Bleiberecht zu gewinnen, wie nicht zuletzt das aktuelle Beispiel des Vereins der Vietnamesen in Cottbus und Umland e.V. zeigt (Verein der Vietnamesen 2020).

\section{FOLLOW THE MEMORY - EINE MULTILO- KALE, POSTKOLONIALE UND POSTSOZIALIS- TISCHE SPURENSUCHE}

Die zuvor genannten Beispiele verdeutlichen, dass Einwanderung in die Region rund um Cottbus eine lange und zugleich komplexe Geschichte hat. Diese kann nicht losgelöst von der kolonialen und sozialistischen Vergangenheit der Stadt verstanden werden. Ferner müssen Verfahren gefunden werden, die dazu beitragen, die Prozesse der Entinnerung, d. h. des aktiven Vergessens von kolonial geprägten Geschichten und Bewegungen sichtbar zu machen (Aikins/Hoppe 2011; Ha, K. N. 2017). Auf diese Weise können Grundlagen für eine kritische Aufarbeitung des kolonialen Erbes geschaffen werden.

Um hegemoniale Formen des Erinnerns und Vergessen aufzubrechen, bieten sich Geschichtswerkstätten oder aktivistische Stadtrundgänge an. Erstere haben im deutschsprachigen Kontext eine längere Tradition in der Aufarbeitung verschiedener Phasen des Nationalsozialismus (Berliner Geschichtswerkstatt 1994). Letztere sind insbesondere im Kontext de- und postkolonialer Initiativen aufgekommen. Diese haben es sich zur Aufgabe gemacht, die Stadtgeschichte hinsichtlich kolonialer Gewalt einerseits und den Möglichkeiten der Dekolonialisierung und des antikolonialen Widerstandes andererseits aufzuarbeiten (Bernhard 2016; Zwischenraum Kollektiv 2017). Beide Ansätze bemühen sich um eine kritische Aufarbeitung einer urbanen Geschichtsschreibung von unten und adressieren Möglichkeiten zur Schaffung epistemischer und sozialer Gerechtigkeit (Castro Varela/Heinemann 2017; Matz et al. 2017).

Das Lehrforschungsprojekt der postkolonialen und postsozialistischen Stadtführung, das in den Sommersemestern 2018 und 2019 an der Brandenburgischen Technischen Universität Cottbus-Senftenberg stattfand, knüpfte an diese Prämissen an. Ziel des Projektes war es, mithilfe der kritischen Aufarbeitung der Geschichten globalgeschichtlich geprägter Einwanderungen einerseits die Kolonialität des städtischen Raumes ( $\mathrm{Ha}$, N. K. 2017) zu beleuchten und andererseits die Realität der Migrationsgesellschaft in der ostdeutschen Stadt aufzuzeigen (Ha 2019). Neben dem Fortwirken kolonialer Episteme ging es um 
die Verwobenheiten der politischen Konfigurationen des Kolonialismus und Sozialismus, die die Stadt nachhaltig prägen und für verschiedene Prozesse der Rassialisierung und des Fremdmachens sorgen. Aufbauend auf post- und dekolonialen Initiativen strebte das Lehrforschungsprojekt an, zu einer Dekolonisierung der Stadt beizutragen, beispielsweise indem es rechtsextremen und rassistischen Narrativen etwas entgegensetzt (Trzeciak/Peters i. E.).

Um Teile der entinnerten Stadtgeschichte(n) sichtbar zu machen und hegemoniale Erinnerungsnarrative zu durchbrechen, führten die Teilnehmer*innen des Lehrforschungsprojektes eine multi-sited ethnography (Marcus 1995) in der Stadt durch. Diese multilokale Form der Ethnographie baut auf den Diskussionen zur writing culture (Clifford/Marcus 1986) auf. Die Mitte der 1980er-Jahre in der Kulturanthropologie geführten Debatten stellten die diskursive Verfasstheit von Wissen und die soziale Konstruktion des Kulturellen heraus. Neben diesen wichtigen Impulsen für das Überdenken essentialistischer Kulturkonzepte haben diese methodologischen Auseinandersetzungen auch zu einer Dekonstruktion des durch die klassische Ethnographie betriebenen othering beigetragen, bei dem nichteuropäische Kulturen als fremd und unterlegen konstruiert wurden. Indem den klassischen Formen der Feldforschung und Wissensproduktion die reflexive Involviertheit der forschenden Person in Kontext und Forschung entgegengehalten wurde, erfuhr die Ethnographie als erste Forschungsstrategie empirischer Sozialforschung „die Ehre einer texttheoretischen Dekonstruktion" (Hirschauer 2001, 429). Im Anschluss daran stellt multi-sited ethnography ein sozialkonstruktivistisches Forschungsprogramm dar, bei dem sich die Forscher*innen auf die Fährten einer kulturellen Formation begeben und sich dabei durch verschiedene soziale und kulturelle Zusammenhänge bewegen (Marcus 1995,
105). Aus dieser Perspektive kann Feldforschung als ein konzeptueller Raum verstanden werden, der durch die Aushandlungen der Akteur*innen konstruiert wird. Diese Leitlinien erweisen sich als gut anschlussfähig an die zuvor dargelegten Befunde der memory studies. So erlaubt es die mobile methodische Vorgehensweise, die "disparaten Entstehungsorte erinnerungskultureller Semantiken und deren Vermittlungswege“ (Spiritova/Götz 2015, 328) in den Blick zu nehmen. Auf diese Weise können zum einen die umkämpften und changierenden Bedeutungen der aufgespürten Erinnerungsorte beleuchtet werden; zum anderen können die unterschiedlichen Positionierungen reflektiert werden, von denen aus die Erinnerungsnarrative bedeutsam werden.

\section{MULTIDIREKTIONALE FORMEN DER ERIN- NERUNG IM POSTKOLONIALEN UND POST- SOZIALISTISCHEN KONTEXT}

Im Rahmen einer kritischen Stadtführung haben wir uns in Cottbus auf die Suche nach postkolonialen und postsozialistischen Erinnerungsorten gemacht. ${ }^{6}$ Aufbauend auf post- und dekolonialen Perspektiven nehmen wir "sowohl den Prozess der Kolonialisierung als auch den einer fortwährenden Dekolonisierung und Rekolonisierung" (Castro Varela/Dhawan 2005, 8) in den Blick und interessieren uns ebenso für materielle wie für epistemische Formen von kolonialer Gewalt respektive der Möglichkeiten des antikolonialen Widerstands. ${ }^{7}$ Koloniale Praktiken umfassen ebenso ein System von Herrschaftsbeziehungen (wie die Besetzung und Ausplünderung von Territorien) wie auch koloniale Repräsentations- und Erkenntnissysteme (Hall 1997). Im Mittelpunkt von Prozessen des othering steht die Imaginierung und Inszenierung eines kolonialen, inferioren Anderen als Gegenbild

\footnotetext{
${ }^{6}$ Erinnerungsorte beziehen sich auf materielle, politische, kulturelle oder imaginäre Topoi. Es geht sowohl um Monumente und Artefakte als auch um symbolische und metaphorische Orte, die ihre „Bezüge und [...] Stellung[en] inmitten sich immer neu formierender Konstellationen und Beziehungen [erhalten] " (François/Schulze 2001, 18; siehe auch Zimmerer 2013, 12). Damit Erinnerungsorte als „zersprengte Fragmente eines verlorenen oder zerstörten Lebenszusammenhanges" (Assmann 2018, 309) Bedeutung erlangen, müssen sie über sprachliche Überlieferung gesichert werden.

7 Während postkoloniale Studien insbesondere in den cultural studies verwurzelt sind und von Diaspora-Wissenschaftler*innen aus dem Nahen Osten und Südasien entwickelt wurden, beruhen dekoloniale Perspektiven stärker auf soziologischen Perspektiven aus dem lateinamerikanischen Raum (Bhambra 2014, 115).
} 
eines überlegenen europäischen Selbst (ElTayeb 2016).

Aufbauend auf postsozialistischen Perspektiven argumentieren wir, dass die sozialen Kontexte Ostdeutschlands nach der Wiedervereinigung nach wie vor von der sozialistischen Vergangenheit politisch, wirtschaftlich und kulturell beeinflusst sind (Stykow 2013, 2). Auch wenn die meisten sozialistischen und kommunistischen Regime politisch ihr Ende gefunden haben, so prägen ihre Spuren weiterhin die Verhältnisse, Praktiken und Wissensformen der Gegenwart (ebd.). Ähnlich wie im Falle des Kolonialismus betrifft dies sowohl die materielle Ebene (wie die Abwicklung der Volkseigenen Betriebe (VEB) oder die Zerstörung von sozialistischem Kulturerbe) als auch die symbolische Ebene (wie die Abwertung ostdeutscher Praktiken und Wissensbestände; Kollmorgen 2011; Heft 2018).

Obwohl Kolonialismus und Sozialismus als zeitliche und politisch verschiedene Hintergründe in die Gegenwart hineinwirken, können sie hinsichtlich ihrer Verschränkungen betrachtet werden. So argumentiert Madina Tlostanova (2012) mit Verweis auf die Sowjetunion und Russland, dass die politischen Konfigurationen des Sozialismus, zwar auf andere Weise als in den westlichen und kapitalistischen Gesellschaften, ebenso in eine koloniale Matrix eingelassen waren. ${ }^{8}$ Auch in der DDR sorgte ein kolonial/modern geprägter Realsozialismus für ein System der sozialen Klassifizierung, das sehr ungleiche Positionierungen, Rechte und soziale Realitäten hervorbrachte (Trzeciak/Peters i. E.). Postkoloniale wie postsozialistische Perspektiven erweisen sich demnach als relevant für den urbanen Raum Cottbus, weil die Stadt sowohl vor, während, als auch nach der DDR in koloniale Kontinuitäten verstrickt war. Gleichzeitig jedoch bargen die realsozialistischen Ideen und Praktiken Potenzial für die Transformation kolonialrassistischer Ordnungen. In der DDR hatte es beispielsweise Umbenennungen von kolonialen und faschistischen Straßenamen gegeben. Zudem hatte die DDR antikoloniale Befreiungsbewegungen unterstützt.

Im Zuge der Spurensuche nach postkolonialen und postsozialistischen Erinnerungsorten rekonstruierten wir verschiedene Erinnerungsorte, wobei das Augenmerk auf den Verflechtungen der sozialhistorischen Konfigurationen im Hinblick auf koloniale (Dis-)Kontinuitäten lag. Die Erarbeitung fand im Zuge von zwei Seminaren in den Sommersemestern 2018 und 2019 statt, die im Rahmen des fächerübergreifenden Studiums von meinem Kollegen Manuel Peters und mir angeboten wurden. Dabei wurden verschiedene Stationen zu dem oben genannten Fokus recherchiert und ausgearbeitet. Zu den Teilnehmenden zählten weiße und PoC-Studierende mit ost- und westdeutscher Herkunft sowie mit und ohne Migrationserfahrungen und -erbe, darunter ein Mitglied des Vereins "Geflüchteten Netzwerk Cottbus e.V.", antifaschistische Aktivist*innen sowie zwei weiße Seniorstudenten, die beide ihr Leben in der Stadt verbracht haben. Die Altersspanne der Seminarteilnehmer*innen bewegte sich zwischen Mitte 20 und 86 Jahren, die Gruppengröße zwischen vier und acht Personen. Als Ergebnis beider Seminare boten wir jeweils eine postkoloniale und postsozialistische Stadtführung an. Die zweite Stadtführung, deren Erfahrungen Ausgangspunkt des vorliegenden Artikels sind, bewarben wir öffentlich über verschiedene soziale Netzwerke und Kanäle sowie über einen Artikel in der Lausitzer Rundschau. Es erschienen insgesamt 46 interessierte Personen. Nachdem wir sechs Erinnerungsorte besucht hatten, luden wir die Gruppe in das Kunstmuseum Dieselkraftwerk Cottbus und reflektierten dort mit ca. 25 Personen den erinnerungspolitischen Rundgang im Hinblick auf gegenwärtige rassistische Kontinuitäten. Aus den Seminaren ist die erinnerungspolitische Initiative "Cottbus Postkolonial und Postsozialistisch" entstanden, die es sich zum Ziel gemacht hat, die Rundgänge sukzessive auszuweiten und regelmäßig anzubieten.

\footnotetext{
8 Der Begriff „koloniale Matrix“ stammt aus der dekolonialen Theorie. Er beschreibt die Verbindung zwischen Modernität/Kolonialität, die durch die Auferlegung einer rassistischen Klassifizierung der Weltbevölkerung im Zuge der Kolonisierung des amerikanischen Kontinents und der Etablierung einer kapitalistischen Arbeitsteilung ab dem 16. Jahrhundert entsteht (Quijano 2000).
} 
Zwei der Erinnerungsorte, das 2007 abgerissene spätsozialistische Gebäude des „Sternchens“ sowie der ehemalige VEB Textilkombinat Cottbus (TKC), in dem auch Vertragsarbeiter*innen beschäftigt waren, möchte ich nun genauer in den Blick nehmen. Daran anschließend zeige ich exemplarisch am Beispiel der Diskussionen während einer Stadtführung im Juli 2019, wie postkoloniale und postsozialistische Kontinuitäten ineinandergreifen und für multidirektionale Formen der Erinnerung sorgen können.

\subsection{Die Zerstörung des sozialistischen Kulturer- bes in der Cottbuser Innenstadt}

Im Stadtzentrum von Cottbus befindet sich eine umzäunte Brachfläche, die für einen umkämpften Schauplatz der Stadt steht. Auf dem verwahrlosten Grundstück hatte zuvor der spätsozialistisch gestaltete Stadtkern gestanden. Im kommunikativen Gedächtnis besonders präsent ist der sternförmige Bau der Mokka- und Milchbar, der damals ein beliebter Alltags- und Freizeitort gewesen war (Krauß 2012). Die Geschichte des "Sternchens", wie das Gebäude umgangssprachlich bezeichnet wurde, steht exemplarisch für die Abwertung des sozialistischen Kulturerbes und die Missachtung ostdeutscher Soziokulturen (Kollmorgen 2011, $325 \mathrm{ff}$.). Das architektonisch und kulturell herausragende Gebäude stand seit Beginn der 1990er-Jahre leer, verfiel und wurde 2007 abgerissen (Krauß 2012). Zugleich verweist die Geschichte des Sternchens auf den politischen Herrschaftsanspruch der DDR. Die futuristische Ästhetik des 1968 erbauten Gebäudes mit seiner sternförmigen, aus sechs Hyparschalen bestehenden Dachkonstruktion stand für eine neue Gesellschaftsform und symbolisierte (real)sozialistischen Fortschritt und Modernität (Trzeciak/Peters i. E.). Der Name „Kosmos“ sowie das weltraumbezogene Interieur der Bar (Krauß 2012) verdeutlichten, dass sich die DDR Ende der 1960er-Jahre zu Zeiten von Mondlandung und Sputnik-Start gewissermaßen einen Platz im Weltall imaginierte. Der Anspruch auf Modernität und Fortschritt, der sich in der Architektur materialisierte, implizierte aber auch ein Streben nach Herrschaft. So imaginierte sich die DDR im Vergleich zu sozialistischen Bruderländern aus dem globalen Süden als fortschrittlicher (Schüle 2003; Trzeciak/Peters i. E.). Über den Verweis auf die eigene Spitzenstellung hinter der Sowjetunion sollte ein nationales Bewusstsein bei der Bevölkerung implementiert werden (Poutrus 2005a, 5).

\subsection{Vertragsarbeit im VEB TKC}

Der VEB TKC, der 1969 gegründet wurde und die bereits bestehende Textilindustrie in der Stadt zentralisierte, kann exemplarisch herangezogen werden, um die Regulierung von Arbeitsmobilität in der DDR im Falle der Leichtindustrie aufzuzeigen (Strnad 2011). Der VEB TKC war der größte Arbeitgeber in Cottbus und einer der größten Textilhersteller in der DDR. Ohne die Arbeitskraft der Vertragsarbeiter*innen hätte das TKC seine Textilproduktion nicht aufrechterhalten können. Anders als in der BRD, in der von 1953 bis 1973 Gastarbeiter*innen angeworben wurden, begann der Einsatz von Arbeitsmigrant*innen in der DDR erst Anfang der 1970erJahre. Insbesondere Näherinnen aus Polen, später Kuba und Vietnam verrichteten die körperlich schwere Arbeit in den Bereichen der Konfektion und der textilen Produktion (ebd.). Die Anwerbung von ausländischen Arbeiter*innen war in den Plänen der DDR zunächst nicht vorgesehen, denn Gastarbeit stellte in den Augen der DDRFunktionär*innen eine Fortführung der NSZwangsarbeit dar (Mende 2013, 152). Allerdings begann der SED-Staat im Zuge des zunehmenden Arbeitskräftemangels und der wirtschaftlichen Krisenerscheinungen die Konditionen für Arbeitsmigration neu auszurichten. Ab den 1980er-Jahren wurden vorwiegend Menschen aus Vietnam und Mosambik angeworben, um den Bedarf zur Erhöhung bzw. Aufrechterhaltung der Produktion zu decken. Anders als die Gastarbeit in Westdeutschland war Arbeitsmigration in der DDR - wenn auch zeitlich begrenzt - als Einsatz vorgesehen, der mit der Möglichkeit auf Ausbildung verbunden und an die allgemein geltenden arbeits- und sozialrechtlichen Bedingungen geknüpft war (ebd., 153). Die Kopplung von Ausbildung und Vertragsarbeit sorgte dafür, dass Arbeitsmigration mit den Politiken der antiimperialistischen Solidarität vereinbart werden konnte (ebd., 156). Dennoch waren die Beziehungen zwischen DDR-Bürger*innen und den Genoss*innen aus anderen sozialistischen Bruderstaaten nicht auf Augenhöhe angelegt. Dies belegen neben den ausbeuterischen Vertrags- 
bedingungen für die Arbeitsmigration aus ehemaligen Kolonialstaaten auch die Vorstellungen von nationaler Differenz und Überlegenheit seitens der DDR-deutschen Kommunist*innen (Poutrus 2005b, 231).

\subsection{Diskussion: Wie sollen die koloniale und so- zialistische Vergangenheit der Stadt erinnert werden?}

Im Zuge der Stadtführung wurde deutlich, dass die Erinnerungsorte aus der jüngeren Stadtgeschichte im kommunikativen Gedächtnis der Bevölkerung verankert und für viele der älteren Besucher*innen mit emotionaler und biographischer Bedeutung verbunden sind. Sie konnten sich mit dem Sternchen identifizieren, denn sie hatten dort in der Vergangenheit Eis gegessen, Kaffee und Cocktails getrunken, in der Disko getanzt und Ausstellungen besucht (Feldnotizen, 16.07.2019). Darüber hinaus stand der Gebäudekomplex rund um das Sternchen für einen prestigeträchtigen Erinnerungsort in Cottbus, der über die Grenzen der Stadt hinaus bekannt war und der die Bedeutung von Cottbus als Zentrum der Energie- und Textilindustrie reflektierte. Der Abriss des Sternchens stellt eine schmerzhafte Erinnerung dar und symbolisiert eine Missachtung des sozialistischen Kulturerbes. Seine Zerstörung nahm den Menschen die Materialität eines konkreten Erinnerungsortes.

Zugleich stellt das Sternchen einen Erinnerungsort dar, an dem sich verschiedene Erinnerungsnarrative überlagern. Einerseits konnten die Folgen und die symbolische sowie materielle Abwertung des DDR-Kulturerbes, die sich auch in den Lebenserfahrungen der Cottbuser Bevölkerung widerspiegelt, zur Sprache kommen (Kollmorgen 2011; Heft 2018). Andererseits kamen, als es um die Aspekte der kolonialen Verstrickungen der sozialistischen Modernität ging, die blinden Flecken der Erinnerung zum Vorschein. Vielen der Teilnehmenden fiel es zunächst schwer, das Überlegenheitsdenken gegenüber anderen sozialistischen Bruderstaaten, das sich in der futuristischen Architektur des Gebäudes widerspiegelte, zu reflektieren.

Nachdem wir mit dem Sternchen einen Erinnerungsort behandelt hatten, der sich mit Abwertung der Lebensgeschichten der weißen und älteren ostdeutschen Teilnehmer*innen befasste und diese anerkannte, schien es einfacher, über die ambivalenten Aspekte der DDR-Geschichte zu sprechen. So thematisierten wir im Anschluss daran die sozialen Realitäten, die durch das Migrationsregime in der DDR produziert worden waren, am Beispiel des VEB Textilkombinats Cottbus (TKC). Die Thematisierung der Ausgrenzung und des Fremdmachens von Vertragsarbeiter*innen entlang einer kolonialen Matrix entfachte eine Debatte darüber, wie die DDR erinnert werden sollte. Nicht alle der weißen Teilnehmer*innen der Stadtführung waren mit der Thematisierung von Rassismus und sozialer Segregation einverstanden, die das DDR-Migrationsregime charakterisiert hatte. Während einige auf die Vertragsbedingungen des Migrationsregimes hinwiesen und die Prozesse der rassistischen Hierarchisierung von Vertragsarbeiter*innen anhand von Erinnerungen aus den VEBs, in denen sie gearbeitet hatten, illustrierten, wiederholten andere das offizielle Narrativ, dass Rassismus in der DDR weniger virulent als in Westdeutschland gewesen sei (Feldnotizen, 16.07.2019). In diesem Kontext betonte ein weiBer Seminarteilnehmer, dass das Leben in der DDR für die Mitglieder der Mehrheitsgesellschaft weniger durch die politischen Imperative des Realsozialismus als durch ein Leben in materieller und sozialer Sicherheit geprägt gewesen war, eine Perspektive, die er im kollektiven Gedächtnis der BRD missrepräsentiert sieht (ebd.). Die konkrete Erinnerung an migrantische Kolleg*innen, die nach 1990 größtenteils in ihre Herkunftsländer zurückkehrten, stellte wiederum einen Raum für transkulturelle Bezüge zur aktuellen Situation von Geflüchteten in Cottbus her. Gleichzeitig blieb es schwierig, die Kontinuitäten von rassistischer Ausgrenzung in der Stadt mit der Verleugnung von Rassismus in der DDR zusammen zu bringen.

Wiederum zeigten die Besucher*innen der Stadtführung weniger Verwunderung, als es um die Spuren der deutschen Kolonialgeschichte in Cottbus ging, die wir anhand von Epitaphien in der Cottbuser Oberkirche für die in kolonialen Kriegen in Deutsch-Südwest-Afrika und China gestorbenen Soldaten illustrierten. Im Gespräch betonten einige der älteren Besucher*innen, die zu DDR-Zeiten in der Stadt gelebt und gearbeitet hatten, dass sie sich während ihrer Schulzeit in 
der DDR kritisch mit der Geschichte des deutschen Imperialismus auseinandergesetzt hatten (Feldnotizen, 16.07.2019). Sie vermuteten, dass sich die offiziellen Erinnerungsnarrative des antiimperialistischen SED-Staates über den deutschen Kolonialismus und Imperialismus wesentlich von denjenigen Westdeutschlands unterschieden (ebd.; siehe auch Ha, K. N. 2017, 114 ff.). Auch war ihnen die Forderung und Notwendigkeit einer dekolonialen Intervention im urbanen Raum nicht neu (Feldnotizen, 16.07.2019).

Deutlich wurde, dass die Verwobenheit zwischen postkolonialen und postsozialistischen Spuren verschiedene Vergangenheitsnarrative zum Vorschein brachte. Je nach sozialer Positionierung rekonstruierten die Besucher*innen der Stadtführung die spezifischen Erinnerungsorte sehr unterschiedlich. Die Kontroverse darüber, wie die kolonialen und sozialistischen Spuren in Cottbus erinnert werden sollten, eröffnete einen produktiven Raum zur Reflexion und Verhandlung weiterer, marginalisierter Geschichten. Über die vergangenheitsbezogenen Dialoge konnten verschiedene soziale Gruppen in Erscheinung treten. So sprachen Mitglieder einer antifaschistischen Initiative während der gemeinsamen Diskussion über die Kontinuitäten des Rassismus sowie rechtsextremer Mobilisierung vor und nach 1989, die sie in den Zusammenhang der derzeitigen Wahlerfolge der AfD stellten (ebd.). Diese Erzählungen nahmen wiederum Mitglieder des „Geflüchteten Netzwerk Cottbus e.V.“ zum Ausgangspunkt, um verschiedene Formen rassistischer Ausgrenzung, aber auch um Formen der Allianz und der Solidarität in der Gegenwart darzulegen (ebd.).

Das konkrete Aufzeigen der spezifischen Prozesse und Positionierungen, von denen aus die koloniale und sozialistische Stadtgeschichte erinnert oder vergessen wurden, verwies auf das umkämpfte Feld der Erinnerungspolitik. Der diskursive Verhandlungsraum war bedeutsam, um einen multidirektionalen Dialog über verschiedene Formen der Erinnerung zu initiieren. So entstand eine Begegnungszone, die verschiedene (asymmetrische) Formen der Ausgrenzung, der Abwertung und des Fremdmachens anerkannte, ohne diese in eine Logik der Konkurrenz zu stellen. Indem aufbauend auf der kritischen
Auseinandersetzung mit dem kolonialen Erbe der Stadt verschiedene multidirektionale Formen des Erinnerns produktiv wurden, konnten Fragen nach sozialer und epistemischer Gerechtigkeit adressiert werden.

Multidirektionale Prozesse des Dialoges in einer Stadt wie Cottbus sind wichtig, um Grundlagen für ein solidarisches Miteinander zwischen verschiedenen, marginalisierten und hegemonialen Gruppen zu schaffen. Auch wenn die Aushandlungen von Erinnerungen nicht notwendigerweise Solidarisierungseffekte mit sich bringen, so können sie einen Beitrag zur Störung und Unterbrechung rechter Narrative sowie Impulse für eine rassismuskritische Sensibilisierung leisten. In diesem Sinne sind erinnerungspolitische Initiativen wie das Stadtführungsprojekt, die kritische Lern- und Reflexionsorte zur Auseinandersetzung mit dem kolonialen Erbe der Gesellschaft anbieten, erst der Anfang von weitreichenderen Maßnahmen der Dekolonisierung von Städten, die nicht nur Fragen der Zugehörigkeit und Teilhabe, sondern auch der Wiedergutmachung betreffen.

\section{Fazit}

In diesem Beitrag bin ich der Frage nachgegangen, wie entinnerte und marginalisierte Geschichten durch dialogische und multiperspektivische Methoden der Erinnerungsarbeit sichtbar gemacht werden können. Aufbauend auf Befunden der dekolonialen und postkolonialen Forschung sowie der memory studies habe ich argumentiert, dass Erinnerungen machtvolle Werkzeuge darstellen, mit denen die Vergangenheit in der Gegenwart bearbeitet und konstruiert wird (Rothberg 2009).

Am Beispiel des Lehrforschungsprojektes einer postkolonialen und postsozialistischen Stadtführung in der ostdeutschen Stadt Cottbus habe ich gezeigt, wie sich Erinnerungen an koloniale und realsozialistische Spuren überlagern und dabei verschiedene Bedeutungen und Konstellationen produzieren. Zwar bringen die Überlagerungen der Hinterlassenschaften von Kolonialismus und Sozialismus ungleiche und diverse Formen der Erinnerung hervor. So scheint die Thematisierung kolonialer Spuren auf den ersten Blick weniger umkämpft als die Verwobenheiten von 
Kolonialismus und Realsozialismus. Wie ich mit Rothberg (ebd.) beleuchtet habe, müssen die umkämpften Formen der Erinnerung jedoch nicht notwendigerweise eine Logik der Konkurrenz um Anerkennung zwischen verschiedenen sozialen Gruppen reproduzieren. Über das Sichtbarmachen verschiedener Erfahrungen des Fremdmachens und der symbolischen und materiellen Missachtung von kollektiven Erfahrungen können auch Formen multidirektionaler Erinnerung entstehen, die das Potenzial für neue Formen der Solidarität und neue Visionen von Gerechtigkeit herstellen. Die kritische Aufarbeitung des Nachwirkens des kolonialen Erbes kann einen Beitrag leisten, um rassistischen und rechtsextremen Mobilisierungen und Narrativen entgegenzuwirken.

\section{LITERATUR}

Aikins, Joshua K./Hoppe, Rosa (2011): Straßennamen. In: Arndt, Susan/Ofuatey-Alazard, Nadja (Hrsg.): Wie Rassismus aus Wörtern spricht: Kerben des Kolonialismus im Wissensarchiv deutsche Sprache. Ein kritisches Nachschlagewerk. Münster: Waxmann, 521538.

Amelina, Anna (2017): After the Reflexive Turn in Migration Studies: Towards the Doing Migration Approach. In: Working Paper Series. Gender, Diversity and Migration, 13. Online verfügbar unter: $\quad$ https://www.fb03.uni-frankfurt.de/67001816/amelina_doing_migration.pdf (14.06.2020).

Assmann, Jan (1999): Kollektives Gedächtnis und kulturelle Identität. In: Assmann, Jan /Hölscher, Tonio (Hrsg.): Kultur und Gedächtnis. Frankfurt am Main: Suhrkamp, 9-19.

Assmann, Aleida (2018): Erinnerungsräume. Formen und Wandlungen des kulturellen Gedächtnisses. München: C. H. Beck.

Attia, Iman/Rothberg, Michael (2018): Multidirectional memory und Verwobene Geschichten. Ein Gedankenaustausch. In: Neue Rundschau, 129 (2), 92-105.

Behrends, Jan/Lindenberger, Thomas/Poutrus, Patrice G. (2003): Fremde und Fremd-Sein in der DDR. Zur Einführung. In: Behrends, Jan/Lindenberger, Thomas/Poutrus, Patrice G. (Hrsg.): Fremde und Fremd-Sein in der DDR. Zu historischen Ursachen der Fremdenfeindlichkeit in Ostdeutschland. Berlin: Metropol, 9-22.

Berliner Geschichtswerkstatt (1994): Alltagskultur, Subjektivität und Geschichte. Zur Theorie und Praxis von Alltagsgeschichte. Münster: Westfälisches Dampfboot.

Bernhard, Philipp (2016): Postkoloniale Spurensuche in München. Geschichtsdidaktische Reflexion eines Stadtrundgangs mit einer ,postkolonialen Initiative' im Rahmen einer koIonialgeschichtlichen Unterrichtseinheit. In: Zeitschrift für Geschichtsdidaktik, 15, 101-115.

Bhambra, Gurminder K. (2014): Postcolonial and decolonial dialogues. In: Postcolonial Studies, 17 (2), 115-121.

Castro Varela, Maria do Mar/Heinemann, Alisha M.B. (2017): „Eine Ziege für Afrika!“ Globales Lernen unter postkolonialer Perspektive. In: Emde, Oliver/Jakubczyk, Uwe/Kappes, Bernd/Overwien, Bernd (Hrsg.): Mit Bildung die Welt verändern? Globales Lernen für eine nachhaltige Entwicklung. Leverkusen: Budrich, 38-54.

Matz, Eugenia/Knake, Sebastian/Garbe, Sebastian (2017): „Gibt's das auch in postkolonial?" Globales Lernen vor dem Hintergrund postkolonialer Kritik. In: Emde, Oliver/Jakubczyk, Uwe/Kappes, Bernd/Overwien, Bernd (Hrsg.): Mit Bildung die Welt verändern? Globales Lernen für eine nachhaltige Entwicklung. Leverkusen: Budrich, 91-109.

Castro Varela, María do Mar/Dhawan, Nikita (2005): Postkoloniale Theorie. Eine kritische Einführung. Bielefeld: transcript.

Clifford, James/Marcus, George E. (Hrsg.) (1986): Writing Culture. The Poetics and Politics of Ethnography. Berkeley: University of California Press.

Confino, Alon/Fritzsche, Peter (2002): Introduction: Noises of the Past. In: Confino, Alon/Fritzsche, Peter (Hrsg.): The Work of Memory. New Directions in the Study of German Society and Culture. Urbana/Chicago: University of Illinois Press, 1-21.

Conrad, Sebastian (2008): Deutsche Kolonialgeschichte. München: C. H. Beck.

Dortmund Postkolonial (2016): Aus dem Kriegsgefangenenlager Cottbus. Online 
verfügbar unter: http://www.dortmund-postkolonial.de/?attachment_id=5237 (14.06.2020).

Eggers, Maureen Maisha/Kilomba, Grada/Piesche, Peggy/Arndt, Susan (Hrsg.): Mythen, Masken und Subjekte - Kritische Weißseinsforschung in Deutschland. Münster: Unrast.

El-Tayeb, Fatima (2016): Undeutsch. Die Konstruktion des Anderen in der postmigrantischen Gesellschaft. Bielefeld: transcript.

François, Etienne/Schulze, Hagen (2001): Einleitung. In: François, Etienne/Schulze, Hagen (Hrsg.): Deutsche Erinnerungsorte, Bd. 1. München: C. H. Beck, 9-26.

Goel, Urmila (2013): Ungehörte Stimmen. Überlegungen zur Ausblendung von Migration in die DDR in der Migrationsforschung. In: Gürsel, Duygu/Çetin, Zülfukar (Hrsg.): Wer MACHT Demo_kratie? Kritische Beiträge zu Migration und Machtverhältnissen. Münster: Edition Assemblage, 138-150.

Ha, Kien Nghi (2017): Die fragile Erinnerung des Entinnerten. In: Zwischenraum Kollektiv (Hrsg.): Decolonize the City! Zur Kolonialität der Stadt. Gespräche, Aushandlungen, Perspektiven. Münster: Unrast, 108-120.

Ha, Noa K. (2017): Zur Kolonialität des Städtischen. In: Zwischenraum Kollektiv (Hrsg.): Decolonize the City! Zur Kolonialität der Stadt. Gespräche, Aushandlungen, Perspektiven. Münster: Unrast, 75-87.

Ha, Noa K. (2019): „Die Realität der ostdeutschen Migrationsgesellschaft wird zu wenig benannt." Interview von Sarah Ulrich. Online verfügbar unter: https://heimatkunde.boell.de/de/2019/11/09/die-realitaet-derostdeutschen-migrationsgesellschaft-wird-zuwenig-benannt (19.10.2020).

Häfner, Daniel/Müller, Bernd (2020): Cottbus befreit! Täter, Opfer, Widerstand im „Dritten Reich“. 2. Auflage. Online verfügbar unter: https://www.dielinke-lausitz.de/fileadmin/lausitz/002_POLITIK/Cottbus/2020/Cottbus_befreit Broschuere.pdf (19.10.2020).

Hall, Stuart (1997): Wann war ,der Postkolonialismus'? Denken an der Grenze. In: Bronfen, Elisabeth/Marius, Benjamin/Steffen, Therese (Hrsg.): Hybride Kulturen. Beiträge zur anglo- amerikanischen Multikulturalismusdebatte. Tübingen: Stauffenberg, 219-246.

Hall, Stuart (1999): Whose Heritage? Un-settling 'The Heritage', Re-imagining the Post-nation. In: Third Text, 13 (49), 3-13.

Harvey, David C. (2001): Heritage Pasts and Heritage Presents: Temporality, Meaning and the Scope of Heritage Studies. In: International Journal of Heritage Studies, 7 (4), 319-338.

Heft, Kathleen (2018): Brauner Osten - Überlegungen zu einem populären Deutungsmuster ostdeutscher Andersheit. In: Feministische Studien, 2 (18), 357-366.

Hirschauer, Stefan (2001): Ethnografisches Schreiben und die Schweigsamkeit des Sozialen. $\mathrm{Zu}$ den Methodologien einer Beschreibung. In: Zeitschrift für Soziologie, 30 (6), 429-451.

Kollmorgen, Raj (2011): Subalternisierung. Formen und Mechanismen der Missachtung Ostdeutscher nach der Vereinigung. In: Kollmorgen, Raj/Koch, Frank T./Dienel, Hans-Liudger (Hrsg.): Diskurse der deutschen Einheit. Kritik und Alternativen. Wiesbaden: VS, 301-359.

Kollmorgen, Raj/Hans, Torsten (2011): Der verIorene Osten. In: Kollmorgen, Raj/Koch, Frank T./Dienel, Hans-Liudger (Hrsg.): Diskurse der deutschen Einheit. Kritik und Alternativen. Wiesbaden: VS, 107-165.

Krauß, Alexander (2012): Aufstieg und Fall der Stadtpromenade Cottbus. In: Freie und Hansestadt Hamburg, Kulturbehörde, Denkmalschutzamt (Hrsg.): Arbeitshefte zur Denkmalpflege in Hamburg, 28, 179-182.

Marcus, George E. (1995): Ethnography in/of the World System: The Emergence of Multi Sited Ethnography. In: Annual Review of Anthropology, 24, 95-117.

Maurin, Jost (2005): Flüchtlinge als politisches Instrument - Chilenische Emigranten in der DDR 1973-1989. In: Totalitarismus und Demokratie, 2. 345-374.

Mende, Christiane (2013): Migration in die DDR. Über staatliche Pläne, migrantische Kämpfe und den real-existierenden Rassismus. In: Gürsel, Duygu/Çetin, Zülfukar/Allmende e. V. (Hrsg.): Wer MACHT Demo_kratie? Kritische Beiträge zu 
Migration und Machtverhältnissen. Münster: Edition Assemblage, 151-164.

Messerschmidt, Astrid (2008): Postkoloniale Erinnerungsprozesse in einer postnationalsozialistischen Gesellschaft. Vom Umgang mit Rassismus und Antisemitismus. In: Peripherie, 109/110 (28), 42-60.

Piesche, Peggy (2002): Black and German? East German Adolescents Before 1989: A Retrospective View of a "Non-Existent Issue" in the GDR. In: Adelson, Leslie A. (Hrsg.): The Cultural AfterLife of East Germany: New Transnational Perspectives. Washington: American Institute for Contemporary German Studies, 37-59.

Piesche, Peggy (Hrsg.) (2019): Labor 89. Intersektionale Bewegungsgeschichte ${ }^{*} n$ aus West und Ost. Berlin: Yilmaz-Günay.

Poutrus, Patrice G. (2005a): Die DDR, ein anderer deutscher Weg? Zum Umgang mit Ausländern im SED-Staat. In: Beier-de Haan, Rosemarie (Hrsg.): Zuwanderungsland Deutschland. Migrationen 1500-2005. Berlin/Wolfratshausen: Deutsches Historisches Museum/Edition Minerva. Online verfügbar unter: https://zeithistorische-

forschungen.de/sites/default/files/medien/material/migrationen_poutrus.pdf (19.10.2020).

Poutrus, Patrice G. (2005b): „Teure Genossen“. Die „politischen Emigranten“ als „Fremde“ im Alltag der DDR-Gesellschaft. In: Poutrus, Patrice G./Müller, Christian T. (Hrsg.): Ankunft - Alltag Ausreise. Migration und interkulturelle Begegnung in der DDR-Gesellschaft. Köln: Böhlau, 221-266.

Quijano, Aníbal (2000): Coloniality of Power and Eurocentrism in Latin America. In: International Sociology, 15 (2), 215-232.

Rothberg, Michael (2009): Multidirectional Memory. Remembering the Holocaust in the Age of Decolonization. Stanford: Stanford University Press.

Rothberg, Michal/Yildiz, Yasemin (2011): Memory Citizenship: Migrant Archives of Holocaust Remembrance in Contemporary Germany. In: Parallax, 17 (4), 32-48.

Schüle, A. (2003): „Die ham se sozusagen aus dem Busch geholt." Die Wahrnehmung der Vertragsarbeitskräfte aus Schwarzafrika und
Vietnam durch Deutsche im VEB Leipziger Baumwollspinnerei. In: Behrends, Jan/Linden berger, Thomas/Poutrus, Patrice G. (Hrsg.): Fremde und Fremd-Sein in der DDR. Zu historischen Ursachen der Fremdenfeindlichkeit in Ostdeutschland. Berlin: Metropol Verlag, 309324.

Schwachenwald, Freya (2019): Art, Nature, Ghosts, and Ice Cream: Transcultural Assemblages of Prince Hermann von Pückler-Muskau (1785-1871) and Machbuba/Ajiamé/Bilillee. In: Journal of Transcultural Studies, 10 (2), 78-120.

Spiritova, Marketa/Götz, Irene (2015): Ethnologische Erkundungen des östlichen Europas am Beispiel der Gedächtnis- und Erinnerungsforschung. In: Götz, Irene/Moser, Johannes/Ege, Moritz/ Lauterbach, Burkhart (Hrsg.): Europäische Ethnologie in München: Ein kulturwissenschaftlicher Reader. Münster: Waxmann, 319-336.

Stadtverwaltung Cottbus (2019): Ergebnisse der Landtagswahl 2019. Online verfügbar unter: https://www.cottbus.de/opt/wahl/Landtagswahl_19_2/LTWZweitstimme.html (14.06.2020).

Strnad, Anja (2011): Vertragsarbeiter in der Leichtindustrie am Beispiel des VEB Textilkombinat Cottbus. In: Priemel, Christian K. (Hrsg.): Transit | Transfer. Politik und Praxis der Einwanderung in der DDR 1945-1990. Berlin: Bebra, 169-187.

Stykow, Petra (2013): Postsozialismus. In: Docupedia-Zeitgeschichte: Begriffe, Methoden und Debatten der zeithistorischen Forschung. Online verfügbar unter: https://docupedia.de/zg/Postsozialismus (14.06.2020).

Theilig, Stephan (2013): Türken, Mohren und Tataren. Soziokulturelle Beziehungen, interkulturelle Transformations- und Translationsprozesse in der Geschichte. Türken, Mohren und Tataren als exotische (Zwangs-)Migranten in Brandenburg-Preußen im 18. Jahrhundert. Berlin: Frank \& Timme.

Tlostanova, Madina (2012): Postsocialist $\neq$ postcolonial? On post-Soviet imaginary and global coloniality. In: Journal of Postcolonial Writing, 48 (2), 130-142. 
Trzeciak, Miriam Friz/Schäfer, Jana (2019): Die Verhandlung von ethnosexueller Zugehörigkeit in Diskursereignissen zu Konflikten um Migration in Cottbus und Köthen. In: Migration und Soziale Arbeit, 3, 8-24.

Trzeciak, Miriam Friz/Peters, Manuel (i. E.): Decolonizing Cottbus: Postcolonial and Postsocialist Entanglements. In: Picker, Giovanni/ Ha, Noa K. (Hrsg.): Provincializing European Cities. Manchester University Press.

Verein Opferperspektive (2019): Keine Sicherheit vor rechter Gewalt. Online verfügbar unter: https://www.opferperspektive.de/aktuelles/keine-sicherheit-vor-rechter-gewalt (14.06.2020).
Verein der Vietnamesen in Cottbus und Umgebung e.V. (2020): Internetauftritt. Online verfügbar unter: http://nguoivietcottbus.de/ (09.11.2020).

Zimmerer, Jürgen (2013): Kolonialismus und kollektive Identität: Erinnerungsorte der deutschen Kolonialgeschichte. In: Zimmerer, Jürgen (Hrsg.): Kein Platz an der Sonne. Erinnerungsorte der deutschen Kolonialgeschichte Frankfurt am Main: Campus-Verlag, 9-38.

Zwischenraum Kollektiv (Hrsg.) (2017): Decolonize the City! Zur Kolonialität der Stadt. Gespräche, Aushandlungen, Perspektiven. Münster: Unrast.

\section{Zum*zur Autor*in}

Miriam Friz Trzeciak ist wissenschaftliche*r Mitarbeiter*in an der BTU Cottbus-Senftenberg. Ihre*seine Forschungsinteressen sind kritische Migrationsforschung, Geschlechterforschung und Queer Theory, Post- und dekoloniale Studien, postsozialistische Studien sowie qualitative und aktivistische/dialogische Forschungsmethoden.

\section{Kontakt}

Dr. Miriam Friz Trzeciak

Fakultät 5 - Lehrstuhl Interkulturalität

Erich-Weinert-Str. 1

LG 10, Raum 206a

D-03046 Cottbus

Tel.: +49355693485

E-Mail: Miriam.Trzeciak@b-tu.de

URL: https://www.b-tu.de/fg-interkulturalitaet/team/akademisches-team/dr-miriam-friz-trzeciak 